\title{
Introduction: \\ Optimizing Outcomes Through Pharmaceutical Advances in the Treatment of Chronic Myeloid Leukemia
}

\author{
David Frame, PharmD
}

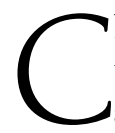
hronic myeloid leukemia (CML) is a cancer of hematopoietic stem cells. It represents approximately $20 \%$ of adult leukemia diagnoses ${ }^{1}$ and affects approximately 1 per 100,000 males and 0.6/0.7 per 100,000 females in the United States. ${ }^{2}$ Although the etiology of CML is poorly understood, it is clear that certain environmental exposures can induce the disease. Benzene exposure has been connected with development of various leukemias, including CML. ${ }^{3}$ The Environmental Protection Agency has regulated benzene exposure, labeling it as a carcinogen and restricting its use; however, benzene is found in many materials, including plastics, rubber, and cigarette smoke, making it difficult to control. Cigarette smoke, in particular, also is associated with increases in myeloid leukemias, as well as increased disease progression for CML. ${ }^{4}$ Ionizing radiation was implicated when cohorts of Japanese survivors of the atomic bombs developed CML, along with acute myelogenous leukemia and acute lymphoblastic leukemia. ${ }^{5}$ The mechanisms by which these environmental exposures lead to CML are poorly understood.

CML is notable because it represents the first cancer to be associated with a specific chromosomal abnormality. In 1960, Nowell and Hungerford reported that white blood cells derived from patients with CML consistently showed the presence of a small aberrant chromosome, termed the Philadelphia chromosome (Ph). ${ }^{6}$ This aberrant chromosome was traced back to the stem cells of individuals with CML and was instrumental in establishing assays for monitoring disease and, ultimately, the first-line therapies used today. It was realized that the $\mathrm{Ph}$ was caused by a translocation of chromosomes 9 and 22.1 When the breakpoints were mapped, it was discovered that a new protein, BCR-ABL, was formed from the joining of the 2 chromosomes. Expression of BCR-ABL in cell culture was found to be sufficient for transformation, and thus BCR-ABL was identified as the putative cause of CML. Today, we use measures of BCR-ABL protein and mRNA as sensitive assays for disease. Drugs that block BCR-ABL function have been developed, and these have proven so efficacious that within a few years of their introduction, they have

\section{J Manag Care Pharm. 2007; 13(8)(Suppl S-a):S2-3}

Copyright@ 2007, Academy of Managed Care Pharmacy. All rights reserved.

\section{Author}

DAVID FRAME, PharmD, is a clinical hematology, oncology, and blood and marrow transplant specialist and an assistant professor of pharmacy, University of Michigan Health System, Ann Arbor, MI. become the front-line therapy for CML.

This supplement covers a symposium held during the Academy of Managed Care Pharmacy's 19th Annual Meeting and Showcase, which was held in San Diego, California, on April 12, 2007. The goal of this symposium was to elucidate how the new BCR-ABL inhibitors have transformed the treatment of CML and how they have affected managed care for this disease state.

The symposium opened with an overview of CML by Jolynn Sessions. She covered the diagnosis criteria for CML and discussed the molecular pathogenesis mediated by BCRABL. Details of the translocation of chromosomes 9 and 22 were described, along with the consequences of breakpoint variation for disease. She covered the clinical course of the disease, observing when BCR-ABL inhibitors are most effective. She described tests used to measure disease, including the costs associated with each; these tests included hematologic, cytogenetic, and polymerase chain reaction assays. Sessions also compared the relative sensitivities of each assay and presented the use and appropriate point to employ each assay.

Christopher Fausel continued with a discussion of imatinib, the current front-line CML therapy, which functions by inhibiting BCR-ABL. Taking a historical approach, he opened with a description of early forms of chemotherapy used to treat CML, beginning with busulfan and hydroxyurea, which were replaced by interferon $\partial$ with or without cytarabine. He then listed the efficacy and adverse effects of these therapies. He also discussed bone marrow transplant and weighed the possibility of cure against the significant possibility of mortality and adverse effects from the transplant for the survivors. The mechanism of action for imatinib was explained, followed by a review of different clinical trials and the results. Finally, Fausel covered the need to monitor imatinib-treated patients with CML, both for adverse effects and for the rising imatinib-resistant clones.

The symposium ended with a presentation by David Frame, in which he discussed imatinib resistance and outlined mechanisms by which it might arise and strategies for avoiding it. He delineated 5 potential mechanisms by which cancer cells can become resistant to imatinib: (1) increased plasma proteins that bind to imatinib, (2) increased efflux of imatinib through active transporters, (3) the arising of mutations in BCR-ABL that block the ability of imatinib to bind, (4) the arising of mutations in the Src pathway, an alternative means of promoting the transformed phenotype, and (5) the 
amplification of the Ph.

Frame continued with how one might define a subtherapeutic dose using clinical trial data. The use of dose escalation was presented, along with several clinical trials that compared increased imatinib dosing to other treatment strategies. In general, data demonstrated that despite the increased number of adverse events, increased imatinib dosing appears to be an effective way to stop resistance and determine outcomes earlier. Frame then discussed dasatinib, a new BCR-ABL inhibitor that is currently in clinical trials. Dasatinib was found to inhibit BCR-ABL, most imatinib-resistant mutations of BCR-ABL, and SRC. Clinical trial data were presented to show how dasatinib could be a useful tool in the struggle to combat drug-resistant CML clones. Frame concluded with a detailed economic analysis for a variety of CML treatment strategies, illustrating that imatinib and dasatinib are cost-effective ways to greatly increase quality-of-life years for patients with CML.

\section{REFERENCES}

\footnotetext{
1. Sawyers CL. Chronic myeloid leukemia. N Engl J Med. 1999; 340:1330-40.

2. Cartwright R. Epidemiology of the chronic leukemias. In: Wiernik PH, Goldman JM, Dutcher JP, Kyle RA, eds. Neoplastic Diseases of the Blood. Cambridge, England: Cambridge University Press; 2003:9-15.

3. Morgan GJ, Alvares CL. Benzene and the hemopoietic stem cell. Chem Biol Interact. 2005;153-154:217-22.

4. Herr R, Ferguson J, Myers N, et al. Cigarette smoking, blast crisis, and survival in chronic myeloid leukemia. Am J Hematol. 1990;34:1-4.

5. Preston DL, Kusumi S, Tomonaga M, et al. Cancer incidence in atomic bomb survivors. Part III. Leukemia, lymphoma and multiple myeloma, 19501987. Radiat Res. 1994;137:S68-S97.

6. Nowell PC, Croce CM. Chromosomal approaches to the molecular basis of
} neoplasia. Symp Fundam Cancer Res. 1986;39:17-29. 